\title{
Weighted Proportional Fair Scheduling for Downlink Nonorthogonal Multiple Access
}

\author{
Marie-Rita Hojeij $\mathbb{D}^{1,2}$ Charbel Abdel Nour $\mathbb{D}^{1}{ }^{1}$ \\ Joumana Farah $\mathbb{D}^{3},{ }^{3}$ and Catherine Douillard $\mathbb{D}^{1}$ \\ ${ }^{1}$ IMT-Atlantique, CNRS UMR 6285 Lab-STICC, UBL, Brest, France \\ ${ }^{2}$ Faculty of Engineering, Holy Spirit University of Kaslik, Jounieh, Lebanon \\ ${ }^{3}$ Faculty of Engineering, Lebanese University, Roumieh, Lebanon \\ Correspondence should be addressed to Charbel Abdel Nour; charbel.abdelnour@imt-atlantique.fr
}

Received 5 January 2018; Revised 28 March 2018; Accepted 12 April 2018; Published 16 May 2018

Academic Editor: Muhammad Z. Shakir

Copyright (C) 2018 Marie-Rita Hojeij et al. This is an open access article distributed under the Creative Commons Attribution License, which permits unrestricted use, distribution, and reproduction in any medium, provided the original work is properly cited.

A weighted proportional fair (PF) scheduling method is proposed in the context of nonorthogonal multiple access (NOMA) with successive interference cancellation (SIC) at the receiver side. The new scheme introduces weights that adapt the classical PF metric to the NOMA scenario, improving performance indicators and enabling new services. The distinguishing value of the proposal resides in its ability to improve long-term fairness and total system throughput while achieving a high level of fairness in every scheduling slot. Finally, it is shown that the additional complexity caused by the weight calculation has only a limited impact on the overall scheduler complexity, while simulation results confirm the claimed improvements, making the proposal an appealing alternative for resource allocation in a cellular downlink system.

\section{Introduction}

Radio access technologies apply multiple access schemes to provide the means for multiple users to access and share resources at the same time. In the 3.9 and fourth generation of mobile communication systems, such as Long-Term Evolution (LTE) [1] and LTE-Advanced [2, 3], orthogonal multiple access (OMA) based on orthogonal frequency division multiplexing (OFDM) and single carrier frequency division multiple access (SC-FDMA) were adopted, respectively, for downlink and uplink transmissions. Orthogonal multiple access techniques have gained their success from their ability to achieve good system-level throughput performance in packet-domain services, while requiring a reasonable complexity, especially due to the absence of multiuser detection.

However, with the proliferation of Internet applications, between the end of 2016 and 2022, total mobile traffic is expected to increase by 8 times [4]. At the same time, communications networks are required to further enhance system efficiency, latency, and user fairness. To this end, nonorthogonal multiple access (NOMA) has recently emerged as a promising candidate for future radio access [5]. By exploiting an additional multiplexing domain, the power domain, NOMA allows the cohabitation of two or more users per subcarrier. User multiplexing is conducted at the transmitter side, on top of the OFDM layer, and multiuser signal separation takes place at the receiver side, using successive interference cancellation (SIC) [6-12].

The main appeal of NOMA is that it improves user fairness while maximizing the total user throughput. The majority of existing works dealing with scheduling in NOMA have investigated and proposed new techniques for improving the system-level performance in terms of system capacity and cell-edge user throughput $[13,14]$.

In [15], throughput performance is assessed for an uplink nonorthogonal multiple access system where optimized scheduling techniques are proposed and evaluated. A cost function is assigned to each possible pair of users, in order to maximize either the sum rate or the weighted sum rate. The user pairing problem is solved by the Hungarian method and 
significant improvements in sum rates and cell-edge rates are shown compared to OMA.

In [16], several new strategies for the allocation of radio resources (in terms of bandwidth and power) in a downlink NOMA system have been investigated and evaluated. The main objective of [16] is to minimize the number of allocated subbands, while guaranteeing a requested service data rate for each user. In this sense, several design issues have been explored: choice of user pairing, subband assignment, optimal and suboptimal power allocation, and dynamic switching to OMA. Simulation results show that the proposed resource allocation techniques provide better performance when NOMA is used, compared to OMA.

In addition to proposing new scheduling techniques for a NOMA-based system, some papers have investigated the commonly used PF scheduler for the good tradeoff it provides between system capacity and user fairness. Several enhancements have been proposed to the PF scheduler in order to further improve the system-level performance of a NOMA system.

In [17], an improved downlink NOMA scheduling scheme based on the PF scheduler is proposed and evaluated. In this sense, modifications to the PF scheduling metric have been introduced in order to improve the fairness at every frame assignment. Results have shown improved performance compared to a NOMA-based system that considers the conventional PF scheduler.

In [11], a weighted PF-based multiuser scheduling scheme is proposed in the context of a nonorthogonal access downlink system for the aim of further enhancing the gain of the cell-edge user. A frequency block access policy is proposed for cell-interior and cell-edge user groups using fractional frequency reuse (FFR), with significant improvements in the user fairness and system frequency efficiency.

Proposing enhancements to the PF scheduling metric has also been investigated in an OMA-based system. Similar to the work done in [11], several papers have proposed weighted versions of the PF scheduler, with the aim of improving user fairness in the OMA context.

In [18], fair weights have been implemented for opportunistic scheduling of heterogeneous traffic types for OMA networks. For designing fair weights, the proposed scheduler takes into account the average channel status as well as resource requirements in terms of traffic types. Simulation analysis demonstrates the efficiency of the proposed scheme in terms of resource utilization and its flexibility with regard to network characteristics changes due to user mobility.

In [19], the problem of fairness deficiency encountered by the PF scheduler when the mobiles experience unequal path loss is investigated. To mitigate this issue, a modified version of the PF scheduler introducing distance compensation factors has been proposed. This solution was shown to achieve both high capacity and high fairness.

In [20], a weighted PF algorithm is proposed in order to maximize best-effort service utility. The reason behind introducing weight factors into the PF metric is to exploit the inherent near-far diversity given by the path loss. The proposed algorithm enhances both best-effort service utility and throughput performance, with a complexity similar to the complexity of the conventional PF scheduler.

Designing fair weights within the PF scheduling metric has shown remarkable improvements in the system's performance of OMA and NOMA-based systems, especially at the level of user fairness.

The above-mentioned studies have shown the advantages of introducing fair weights within the PF scheduler, especially when combined with a NOMA system. However, increasing the achieved user rate at every slot has not been tackled in these studies. Indeed, such a feature can have a positive impact on the perceived quality of service (QoS), especially for multimedia services on one side, and can reduce the amount of required buffering and memory at the user terminal on the other side. Accordingly, we aim in this paper to combine the advantages of NOMA in terms of spectral efficiency with an implementation of fair weights at the scheduling level in order to improve both the achieved user rate and the user fairness at each time slot. We propose indeed a weighted PF metric where several designs of the introduced weights are evaluated. The proposed scheme aims at providing fairness among users for each channel realization. By doing so, not only is short-term fairness achieved but also user capacity and long-term fairness are enhanced accordingly. On the other hand, the proposed schemes mitigate the problem of zero-rate incidence, inherent to PF scheduling, by attempting to provide nonzero rate to each user in any time scale of interest. This will further enhance the quality of experience (QoE) of all users.

This paper is organized as follows. In Section 2, we introduce the system model and give a general description of the NOMA-based PF scheduler. Section 3 details the proposed weighted schemes in the NOMA context. In Section 4, we apply the fair weights to a resource allocation system based on OMA. Section 5 describes a specific treatment to be applied to the first time slot in order to further enhance fairness. In Section 6, we propose some changes to the weighted metrics in order to give the possibility of delivering different levels of quality of service. Simulation results are given and analyzed in Section 7, while Section 8 concludes the paper.

\section{System Description}

2.1. Basic NOMA System. In this section, we describe the basic concept of NOMA including user multiplexing at the transmitter of the base station (BS) and signal separation at the receiver of the user terminal.

In this paper, a downlink system with a single input single output ( $\mathrm{SISO}$ ) antenna configuration is considered. The system consists of $K$ users per cell, with a total bandwidth $B$ divided into $S$ subbands.

Among the $K$ users, a subset of users $U_{s}=\left\{k_{1}, k_{2}, \ldots\right.$, $\left.k_{n}, \ldots, k_{n(s)}\right\}$ is selected to be scheduled over each frequency subband $s(1 \leq s \leq S)$. The $n$th user $(1 \leq n \leq n(s))$ scheduled at subband $s$ is denoted by $k_{n}$, and $n(s)$ indicates the number of users nonorthogonally scheduled at subband $s$. At the BS transmitter side, the information sequence of each scheduled user at subband $s$ is independently coded and modulated, resulting in symbol $x_{s, k_{n}}$ for the $n$th scheduled user. Therefore, 
the signal transmitted by the BS on subband $s, x_{s}$, represents the sum of the coded and modulated symbols of the $n(s)$ scheduled users:

$$
x_{s}=\sum_{n=1}^{n(s)} x_{s, k_{n}}, \quad \text { with } E\left[\left|x_{s, k_{n}}\right|^{2}\right]=P_{s, k_{n}},
$$

where $P_{s, k_{n}}$ is the power allocated to user $k_{n}$ at subband $s$. The received signal vector of user $k_{n}$ at subband $s, y_{s, k_{n}}$, is represented by

$$
y_{s, k_{n}}=h_{s, k_{n}} x_{s, k_{n}}+w_{s, k_{n}}
$$

where $h_{s, k_{n}}$ is the channel coefficient between user $k_{n}$ and the BS at subband s. $w_{s, k_{n}}$ represents the received Gaussian noise plus intercell interference experienced by user $k_{n}$ at subband $s$. Let $P_{\max }$ be the maximum allowable power transmitted by the BS. Hence, the sum power constraint is formulated as follows:

$$
\sum_{s=1}^{S} \sum_{n=1}^{n(s)} P_{s, k_{n}}=P_{\max }
$$

The SIC process [21] is conducted at the receiver side, and the optimal order for user decoding is in the increasing order of the channel gains observed by users, normalized by the noise and intercell interference $h_{s, k_{n}}^{2} / n_{s, k_{n}}$, where $n_{s, k_{n}}$ is the average power of $w_{s, k_{n}}$. Therefore, any user can correctly decode the signals of other users whose decoding order comes before that user. In other words, user $k_{n}$ at subband $s$ can remove the interuser interference from the $j$ th user, $k_{j}$, at subband $s$, provided $h_{s, k_{j}}^{2} / n_{s, k_{j}}$ is lower than $h_{s, k_{n}}^{2} / n_{s, k_{n}}$, and it treats the received signals from other users with higher $h_{s, k_{j}}^{2} / n_{s, k_{j}}$ as noise $[7,22]$.

Assuming successful decoding and no error propagation and supposing that intercell interference is randomized such that it can be considered as white noise $[9,15]$, the throughput of user $k_{n}$ at subband $s, R_{s, k_{n}}$, is given by

$$
\begin{aligned}
R_{s, k_{n}} & =\frac{B}{S} \log _{2}(1 \\
+ & \left.\frac{h_{s, k_{n}}^{2} P_{s, k_{n}}}{\sum_{j \in N_{s}, h_{s, k_{n}}^{2} / n_{s, k_{n}}<h_{s, k_{j}}^{2} / n_{s, k_{j}} h_{s, k_{n}}^{2} P_{s, k_{j}}+n_{s, k_{n}}}}\right) .
\end{aligned}
$$

It should be noted that most of the papers dealing with resource allocation in downlink NOMA [10, 22-24] consider a maximum number of users per subband of two in order to limit the SIC complexity in the mobile receiver, except for [9] and [25], where this number, respectively, reaches 3 and 4 . However, in the last two cases, static power allocation is assumed, which simplifies the power allocation step but degrades throughput performance. It has also been stated that the performance gain obtained with 3 or 4 users per subband is minor in comparison to the case with 2 users.
2.2. Conventional PF Scheduling Scheme. The PF scheduling algorithm has been proposed to ensure balance between cell throughput and user fairness. Kelly et al. [26] have defined the proportional fair allocation of rates and used a utility function to represent the degree of satisfaction of allocated users. In [27], the practical implementation of the PF scheduler is detailed: at the beginning of every scheduling slot, each user provides the base station with its channel state (or equivalently its feasible rate). The scheduling algorithm keeps track of the average throughput $T_{k}(t)$ of each user in a past window of length $t_{c}$. In the scheduling slot $t$, user $k^{*}$ is selected to be served based on [27]

$$
k^{*}=\arg \max _{k} \frac{R_{k}(t)}{T_{k}(t)},
$$

where $R_{k}(t)$ is the feasible rate of user $k$ for scheduling slot $t$.

In [28], an approximated version of the PF scheduler for multiple users transmission is presented. This version has been adopted in the majority of the works dealing with NOMA [22, 23, 25] in order to select users to be nonorthogonally scheduled on available resources.

For a subband $s$ under consideration, the PF metric is estimated for each possible combination of users $U$, and the combination that maximizes the PF metric is denoted by $U_{s}$ :

$$
U_{s}=\underset{U}{\operatorname{argmax}} \sum_{k \in U} \frac{R_{s, k}(t)}{T_{k}(t)} .
$$

$R_{s, k}(t)$ denotes the instantaneous achievable throughput of user $k$ at subband $s$ and scheduling time slot $t$.

Note that the total number of combinations tested for each considered subband is

$$
N_{U}=\left(\begin{array}{l}
K \\
1
\end{array}\right)+\left(\begin{array}{l}
K \\
2
\end{array}\right)+\cdots+\left(\begin{array}{c}
K \\
N(s)
\end{array}\right) .
$$

Equation (7) represents the general formulation of the case, where a maximum of $N(s)$ users are to be multiplexed in the power domain. In this case, all the combinations of possible users up to $N(s)$ should be verified via the PF metric and the particular user combination leading to the best PF metric value should be chosen for allocation.

$R_{s, k}(t)$ is calculated based on (4), whereas $T_{k}(t)$ is recursively updated as follows [28]:

$$
T_{k}(t+1)=\left(1-\frac{1}{t_{c}}\right) T_{k}(t)+\frac{1}{t_{c}} \sum_{s=1}^{S} R_{s, k}(t) .
$$

Parameter $t_{c}$ defines the throughput averaging time window. In other words, this is the time horizon in which we want to achieve fairness. $t_{c}$ is chosen to guarantee a good tradeoff between system performance (in terms of fairness) and system capacity. We assume in the following a $t_{c}$ window of 100 time slots. With a time slot duration equal to $1 \mathrm{~ms}$, a $100 \mathrm{~ms}$ average user throughput $T_{k}(t)$ is therefore considered. 


\section{Proposed Weighted NOMA-Based Proportional Fairness (WNOPF) Scheduler}

The PF scheduler aims at both achieving high data rates and ensuring fairness among users, but it only considers longterm fairness. In other words, a duration of $t_{c}$ time slots is needed to achieve fairness among users. However, short-term fairness and fast convergence towards required performance are an important issue to be addressed in upcoming mobile standards [4].

Since all possible combinations of candidate users are tested for each subband, a user might be selected more than once and attributed multiple subbands during the same time slot. On the other hand, it can also happen that no subband and therefore no transmission rate are allocated for multiple scheduling slots to a user with a high historical rate. This behavior can be very problematic in some applications, especially those requiring a quasi-constant QoE such as multimedia transmissions. In such cases, buffering may be needed. However, such a scenario may not be compatible with applications requiring low latency transmission.

Therefore, we propose several weighted PF metrics that aim at

(i) enhancing the user capacity, thus increasing the total achieved user throughput;

(ii) reducing the convergence time towards required fairness performance;

(iii) enhancing fairness among users (both long-term and short-term fairness);

(iv) limiting the fluctuations of user data rates;

(v) incorporating the delivery of different levels of QoS.

The proposed scheduler consists of introducing fair weights into the conventional PF scheduling metric. The main goal of the weighted metrics is to ensure fairness among users in every scheduling slot.

To do so, we start by modifying the PF metric expression so as to take into account the status of the current assignment in time slot $t$. Therefore, the scheduling priority given for each user is based not only on its historical rate but also on its current total achieved rate (throughput achieved during the current scheduling slot $t$ ), as proposed in [17].

Scheduling is performed subband by subband and on a time slot basis. For each subband $s$, the conventional PF metric $\mathrm{PF}_{s}^{\mathrm{NOMA}}$ and a weight factor $W(U)$ are both calculated for each candidate user set $U$. Then, the scheduler selects the set of scheduled users $U_{s}$ which maximizes the weighted metric $\operatorname{PF}_{s}^{\mathrm{NOMA}}(U) \times W(U)$. The corresponding scheduling method is referred to as weighted NOMA PF scheduler, denoted by $\mathrm{WPF}^{\mathrm{NOMA}}$. The resource allocation metric can be formulated as follows:

$$
\begin{aligned}
\mathrm{WPF}_{s}^{\mathrm{NOMA}}(U) & =\mathrm{PF}_{s}^{\mathrm{NOMA}}(U) \times W(U) \\
U_{s} & =\underset{U}{\operatorname{argmax}} \mathrm{WPF}_{s}^{\mathrm{NOMA}}(U) .
\end{aligned}
$$

The proposed weight calculation for each candidate user set $U$ relies on the sum of the weights of the multiplexed users.

$$
W(U)=\sum_{k \in U} W_{k}(t)
$$

with

$$
W_{k}(t)=R_{\mathrm{avg}}^{e}(t)-R_{k}(t), \quad k \in U .
$$

$R_{\mathrm{avg}}^{e}(t)$ is the expected achievable bound for the average user data rate in the current scheduling slot $t$. It is calculated as follows:

$$
R_{\mathrm{avg}}^{e}(t)=b \cdot R_{\mathrm{avg}}(t-1)
$$

Since we tend to enhance the achieved user rate in every slot, each user must target a higher rate compared to the rate previously achieved. Therefore, parameter $b$ is chosen to be greater than 1 .

The average user data rate, $R_{\text {avg }}(t)$, used in (12), is updated at the end of each scheduling slot based on the following:

$$
R_{\mathrm{avg}}(t)=\frac{1}{K} \sum_{k=1}^{K} \sum_{s=1}^{S} R_{s, k}(t),
$$

where $R_{s, k}(t)$ is the data rate achieved by user $k$ on subband $s$.

On the other hand, $R_{k}(t)$, the actual achieved data rate by user $k$ during scheduling slot $t$, is calculated as

$$
R_{k}(t)=\sum_{s \in S_{k}} R_{s, k}(t), \quad k \in U
$$

with $S_{k}$ being the set of subbands allocated to user $k$ during time slot $t$. At the beginning of every scheduling slot, $S_{k}$ is emptied; each time user $k$ is being allocated a new subband, and $S_{k}$ and $R_{k}(t)$ are both updated.

The main idea behind introducing weights is to minimize the rate gap among scheduled users in every scheduling slot, thus maximizing fairness among them. A user set $U$ is provided with a high priority among candidate user sets if it contains nonorthogonally multiplexed users experiencing a good channel quality on subband $s$, having low or moderate historical rates, or/and having large rate distances between multiplexed users' actual achieved rates and their expected achievable average user throughput. The highest level of fairness is achieved when all users reach the expected user average rate $R_{\mathrm{avg}}^{e}(t)$. By applying the proposed scheduling procedure, we aim to enhance long-term and short-term fairness at the same time.

It was shown in [28] that the scheduling metric $\mathrm{PF}^{\mathrm{NOMA}}$, defined in (6), strikes a good tradeoff between throughput and fairness, since it maximizes the sum of users service utility which can be formally written as

$$
\mathrm{PF}^{\mathrm{NOMA}}=\max _{\text {scheduler }} \sum_{k=1}^{K} \log T_{k} .
$$

Therefore, any enhanced scheduling metric like WNOPF can strike a better throughput-fairness balance (by achieving 
a higher service utility), compared to the conventional PF scheduler, provided that

$$
\sum_{k=1}^{K} \log T_{k} \geq \sum_{k=1}^{K} \log T_{k}^{\prime}
$$

where the historical rates $T_{k}$ and $T_{k}^{\prime}$ correspond to the schedulers using the WNOPF metric and the conventional PF metric, respectively.

Inspired by the work in [20], in the sequel, we show how this goal can be achieved by an appropriate design of the weights which verifies the constraints provided in Proposition 1.

Proposition 1. To make (16) valid, for a NOMA-based system, the following inequality should be verified:

$$
\prod_{k=1}^{K} W\left(U_{k}\right) \prod_{k=1}^{K} E\left[R_{s, k}\right] \geq \prod_{k=1}^{K} E\left[R_{s, k}^{\prime}\right] .
$$

$E\left[R_{s, k}\right]$ and $E\left[R_{s, k}^{\prime}\right]$ are the statistical average of the instantaneous transmittable rate of user $k$ on a subband $s$, when $W N O P F$ and the conventional PF scheduler are applied, respectively. $U_{k}$ denotes a scheduled user set containing user $k$, $U$ is a possible candidate user set, and $W\left(U_{k}\right)$ is the weight of the set $U_{k}$.

Proof. Equation (16) can be written as

$$
\prod_{k=1}^{K} T_{k} \geq \prod_{k=1}^{K} T_{k}^{\prime}
$$

If we consider that $T_{k}=I_{k \text {,tot }} /\left(t_{c} \Delta T\right)$, where $I_{k \text {,tot }}$ is the total amount of information that can be received by user $k$, for a total observation time $t_{c} \Delta T$, and $\Delta T$ is the scheduling time slot length, we obtain

$$
\prod_{k=1}^{K} \frac{I_{k, \text { tot }}}{t_{c} \Delta T} \geq \prod_{k=1}^{K} \frac{I_{k, \text { tot }}^{\prime}}{t_{c} \Delta T} .
$$

If we denote by $N_{k}$ the number of allocated time slots for user $k$ within $t_{c}$ and by $n_{k}$ the statistical average of the number of allocated subbands to user $k$ per time slot, (19) can be rewritten as

$$
\prod_{k=1}^{K} \frac{N_{k} n_{k} E\left[R_{s, k}\right] \Delta T}{t_{c} \Delta T} \geq \prod_{k=1}^{K} \frac{N_{k}^{\prime} n_{k}^{\prime} E\left[R_{s, k}^{\prime}\right] \Delta T}{t_{c} \Delta T} .
$$

Using a simple rearrangement, we get

$$
\frac{\prod_{k=1}^{K}\left(N_{k} / t_{c}\right) S\left(n_{k} / S\right)}{\prod_{k=1}^{K}\left(N_{k}^{\prime} / t_{c}\right) S\left(n_{k}^{\prime} / S\right)} \geq \frac{\prod_{k=1}^{K} E\left[R_{s, k}^{\prime}\right]}{\prod_{k=1}^{K} E\left[R_{s, k}\right]} .
$$

If $\operatorname{Pr}_{k}\left(=N_{k} / t_{c}\right)$ denotes the probability of user $k$ being scheduled per time slot and $\operatorname{pr}_{k}\left(=n_{k} / S\right)$ denotes the probability of user $k$ being scheduled per subband, (21) can be reformulated as

$$
\frac{\prod_{k=1}^{K} \operatorname{Pr}_{k} \mathrm{pr}_{k}}{\prod_{k=1}^{K} \operatorname{Pr}_{k}^{\prime} \operatorname{pr}_{k}^{\prime}} \geq \frac{\prod_{k=1}^{K} E\left[R_{s, k}^{\prime}\right]}{\prod_{k=1}^{K} E\left[R_{s, k}\right]} .
$$

$\mathrm{pr}_{k}$ can be regarded as the probability of a set $U_{k}$, that is, $\operatorname{Pr}\left(U_{k}\right)$, being chosen among all possible candidate sets $U$ to be scheduled per subband.

Let us consider two sets of users $U_{1}$ and $U_{2}$. If the probability of the user set $U_{1}$ is greater than that of $U_{2}, U_{1}$ will be chosen to be scheduled. Equivalently, the corresponding scheduling metric for user set $U_{1}$ will be in this case larger than that of $U_{2}$. Therefore, in fact, a user set is chosen if the corresponding PF metric is the largest.

Thus, (22) can be equivalent to the following equation:

$$
\frac{\prod_{k=1}^{K} \operatorname{Pr}_{k} \operatorname{PF}^{\mathrm{NOMA}}\left(U_{k}\right) W\left(U_{k}\right)}{\prod_{k=1}^{K} \operatorname{Pr}_{k}^{\prime} \mathrm{PF}^{\mathrm{NOMA}}\left(U_{k}\right)} \geq \frac{\prod_{k=1}^{K} E\left[R_{s, k}^{\prime}\right]}{\prod_{k=1}^{K} E\left[R_{s, k}\right]} .
$$

Note that, in a NOMA-based system, the probability of a user being scheduled per time slot remains the same when using the proposed weighted metric or the conventional PF metric, since users are distributed with uniform and random probability over the entire network in each time slot. Thus, we adopt the following approximation:

$$
\operatorname{Pr}_{k} \simeq \operatorname{Pr}_{k}^{\prime} \text {. }
$$

Additional observations and verifications related to this approximation are given in Section 7. Therefore, (23) and (24) can also be formulated as (17).

Other configurations of rate-distance weights can also be introduced. A promising one is obtained by substituting (25) for (9) and (10):

$$
U_{s}=\arg \max _{U} \sum_{k \in U} \frac{R_{s, k}(t)}{T_{k}(t)} W_{k}(t), \quad k \in U .
$$

Here, the conventional NOMA-based PF metric and the weights are jointly calculated for each user $k$ in candidate user set $U$. By doing so, we assign to each user its weight while ignoring the cross effect $\left(R_{s, k \mid U}(t) / T_{k \mid U}(t)\right) W_{k^{\prime} \mid U}(t)$ produced by (9), where $k$ and $k^{\prime}$ are nonorthogonally multiplexed users in the same $U$. This joint-based incorporation of weights is denoted by J-WNOPF in the following evaluations.

\section{Proposed Weighted OMA-Based PF Scheduler (WOPF)}

In the majority of existing works dealing with fair scheduling, OMA-based systems are considered. For this reason, we propose applying the weighted proportional fair scheduling metric introduced in this paper to an OMA-based system as well. This allows the contribution of NOMA within our framework to be evaluated. In the OMA case, nonorthogonal cohabitation is not allowed. Instead, a subband $s$ is allocated to only one user, based on the following metric:

$$
k^{*}=\arg \max _{k} \frac{R_{s, k}(t)}{T_{k}(t)} W_{k}(t),
$$

where $W_{k}(t)$ is the weight assigned to user $k$, calculated similarly to the weights in WNOPF. The conventional OMAbased $\mathrm{PF}$ scheduling metric is denoted by $\mathrm{PF}^{\mathrm{OMA}}$, whereas 
the resulting scheduling algorithm combining OMA with the proposed weighted PF is denoted by WOPF.

OMA can be regarded as a special case of NOMA, where only one user is allowed to be scheduled per subband. Therefore, in order to achieve a higher user service utility with WOPF than with the conventional PF scheduler in OMA, Proposition 1, detailed and proven in Section 3, should also be verified for an OMA-based system. For this purpose, (17) is modified as follows:

$$
\prod_{k=1}^{K} W_{k} \prod_{k=1}^{K} E\left[R_{s, k}\right] \geq \prod_{k=1}^{K} E\left[R_{s, k}^{\prime}\right],
$$

where $W_{k}$ is the weight assigned to user $k$.

Note that, as in the NOMA case, we assume that the probability of a user being scheduled per time slot remains the same when using the proposed weighted metric or the conventional PF metric.

\section{Proposed Scheduling Metric for the First Scheduling Slot}

In the first scheduling slot, the historical rates and the expected user average data rate are all set to zero. Hence, the selection of users by the scheduler is only based on the instantaneous achievable throughputs. Therefore, fairness is not achieved in the first scheduling slot, and the following slots are penalized accordingly. To counteract this effect, we propose treating the first scheduling slot differently, for all the proposed weighted metrics.

For each subband $s$, the proposed scheduling process selects $U_{s}$ among the candidate user sets based on the following criterion:

$$
U_{s}=\arg \max _{U} \sum_{k \in U} \frac{R_{s, k}(t=1)}{R_{k}(t=1)} .
$$

Note that when WOPF is considered, the maximum number of users per set $U$ is limited to 1 .

$R_{k}(t=1)$, the actual achieved throughput, is updated each time a subband is allocated to user $k$ during the first scheduling slot. By doing so, we give priority to the user experiencing a good channel quality with regard to its actual total achieved data rate, thus enhancing fairness in the first slot.

\section{Incorporation of Premium Services}

In this section, we propose some changes to the proposed weighted metrics in order to give the possibility of delivering different levels of quality of service. In other words, the proposed metrics should have the ability to provide different priorities to different users or to guarantee a certain level of performance to a data flow. To do so, (11) is modified as follows:

$$
W_{k}(t)=R_{\text {service }}-R_{k}(t), \quad k \in U,
$$

where $R_{\text {service }}$ is the data rate requested by a certain group of users, corresponding to a certain level of performance.
As an example, we detail an example of 3 services, although the proposed modifications can be applied to an arbitrary number of services. $R_{\text {service }}$ is then defined as follows:

$$
R_{\text {service }}= \begin{cases}R_{\text {basic }}, & \text { if } k \text { requests a basic service } \\ R_{\text {silver }}, & \text { if } k \text { requests a silver service } \\ R_{\text {gold }}, & \text { if } k \text { requests a gold service, }\end{cases}
$$

$k \in U$.

This modification aims to guarantee a minimum requested service data rate for each user and also tends to enhance the overall achieved fairness between users belonging to the same group, that is, asking for the same service.

\section{Numerical Results}

7.1. System Model Parameters and Performance Evaluation. This subsection presents the system level simulation parameters used to evaluate the proposed scheduling techniques. The parameters considered in this work are based on existing LTE/LTE-Advanced specifications [29]. We consider a baseline SISO antenna configuration. The maximum transmission power of the base station is $46 \mathrm{dBm}$. The system bandwidth is $10 \mathrm{MHz}$ and is divided into 128 subbands when not further specified. The noise power spectral density is $4 \cdot 10^{-18} \mathrm{~mW} / \mathrm{Hz}$. Users are deployed randomly in the cell and the cell radius is set to $500 \mathrm{~m}$. Distance-dependent path loss is considered with a decay factor of 3.76. Extended typical urban (ETU) channel model is assumed, with time-selectivity corresponding to a mobile velocity of $50 \mathrm{~km} / \mathrm{h}$, at the carrier frequency of $2 \mathrm{GHz}$. In both OMA and NOMA scenarios, equal repartition of power is considered among subbands, as considered in $[9,23,24]$. In the case of NOMA, fractional transmit power allocation (FTPA) [30] is used to allocate power among scheduled users within a subband. Without loss of generality, NOMA results are shown for the case where the maximum number of scheduled users per subband is set to $2(n(s)=2)$.

As for parameter $b$ in (12), after several tests, the best performance was observed for $b$ equal to 1.5 . In fact, the system has a rate saturation bound with respect to parameter $b$, since when we further increase $b$, similar performance is maintained.

7.2. Performance Evaluation. In this part, we mainly consider four system-level performance indicators: achieved system capacity, long-term fairness, short-term fairness, and celledge user throughput.

Several techniques are evaluated and compared. The following acronyms are used to refer to the main studied methods:

(i) $\mathrm{PF}^{\mathrm{NOMA}}$ : conventional $\mathrm{PF}$ scheduling metric in a NOMA-based system

(ii) WNOPF: proposed weighted PF scheduling metric in a NOMA-based system

(iii) J-WNOPF: proposed weighted PF scheduling metric with a joint incorporation of weights in a NOMAbased system 
(iv) $\mathrm{PF}_{\text {modified }}^{\mathrm{NOMA}}$ : a modified version of the PF scheduling metric proposed in [17], where the actual assignment of each frame is added to the historical rate

(v) $\mathrm{PF}^{\mathrm{OMA}}$ : conventional $\mathrm{PF}$ scheduling metric in an OMA-based system

(vi) WOPF: proposed weighted PF scheduling metric in an OMA-based system

In order to assess the fairness performance achieved by the different techniques, a fairness metric needs to be defined first. Gini fairness index [31] measures the degree of fairness that a resource allocation scheme can achieve. It is defined as

$$
G=\frac{1}{2 K^{2} \bar{r}} \sum_{x=1}^{K} \sum_{y=1}^{K}\left|r_{x}-r_{y}\right|
$$

with

$$
\bar{r}=\frac{\sum_{k=1}^{K} r_{k}}{K} .
$$

$r_{k}$ is the throughput achieved by user $k$. When long-term fairness is evaluated, $r_{k}$ is considered as the total throughput achieved by user $k$ averaged over a time-window length $t_{c}$ :

$$
r_{k}=\frac{1}{t_{c}} \sum_{t=1}^{t_{c}} R_{k}(t) .
$$

Otherwise, when fairness among users is to be evaluated within each scheduling slot, short-term fairness is considered and $r_{k}$ is taken equal to $R_{k}(t)$, the actual throughput achieved by user $k$ during scheduling slot $t$.

Gini fairness index takes values between 0 and 1, where $G=0$ corresponds to the maximum level of fairness among users, while a value of $G$ close to 1 indicates that the resource allocation scenario is highly unfair.

First, we check the validity of Proposition 1 detailed in Sections 3 and 4 and the validity of the assumption done in (24). Figure 1 shows the observed ratio between $\operatorname{Pr}_{k}$ and $\operatorname{Pr}_{k}^{\prime}$, denoted by Ratio 1, for different values of the number of users per cell. Figure 1 also shows the ratio between the left-hand and the right-hand expressions of (17), denoted by Ratio 2 . Results show that Ratio 1 is very close to 1 , which means that the probability of a user being scheduled per time slot remains the same under the proposed weighted metric or under the conventional PF metric. In addition, Ratio 2 is shown to be greater than 1 regardless of the number of users per cell, which verifies Proposition 1, defined in (17). The results of a similar verification for an OMA system are observed in Figure 2.

Figure 3 shows the system capacity achieved with each of the simulated methods for different numbers of users per cell. Curves in solid lines represent the NOMA case, whereas curves with dotted lines refer to OMA.

We can observe that the throughput achieved with all the simulated methods increases as the number of users per cell is increased, even though the total number of used subbands is constant. This is due to the fact that the higher the number of users per cell is, the better the multiuser diversity is exploited by the scheduling scheme, as also observed in [18].
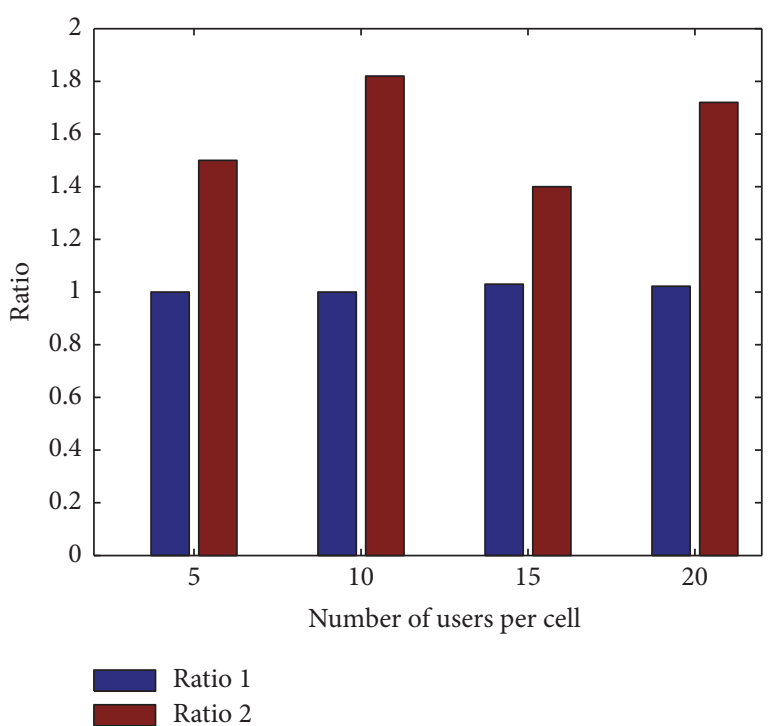

FIgURE 1: Observed ratios related to (17) and (26) versus number of users per cell, NOMA-based system.

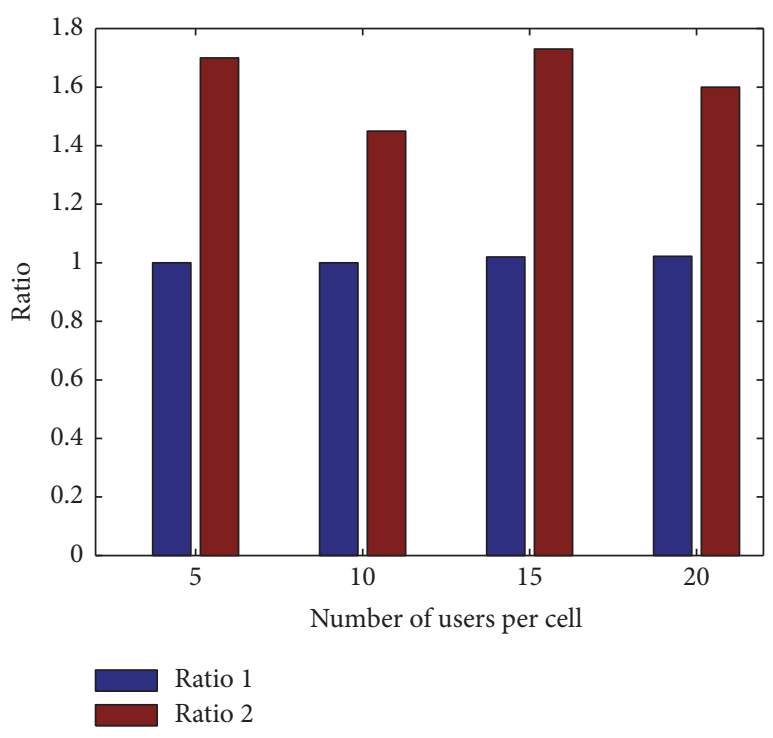

FIGURE 2: Observed ratio related to (26) and (29) versus number of users per cell, OMA-based system.

The gain achieved by WNOPF, when compared to the other proposed weighted metric J-WNOPF, is mainly due to the fact that the joint incorporation of weights does not take into consideration the cross effect produced by nonorthogonally multiplexed users.

The gain in performance obtained by the introduction of weights in the scheduling metric, compared to the conventional $\mathrm{PF}^{\mathrm{NOMA}}$ metric, stems from the fact that, for every channel realization, the weighted metrics try to ensure similar rates to all users, even those experiencing bad channel conditions. With $\mathrm{PF}^{\mathrm{NOMA}}$, such users would not be chosen frequently, whereas appropriate weights give them a higher chance to be scheduled more often. 


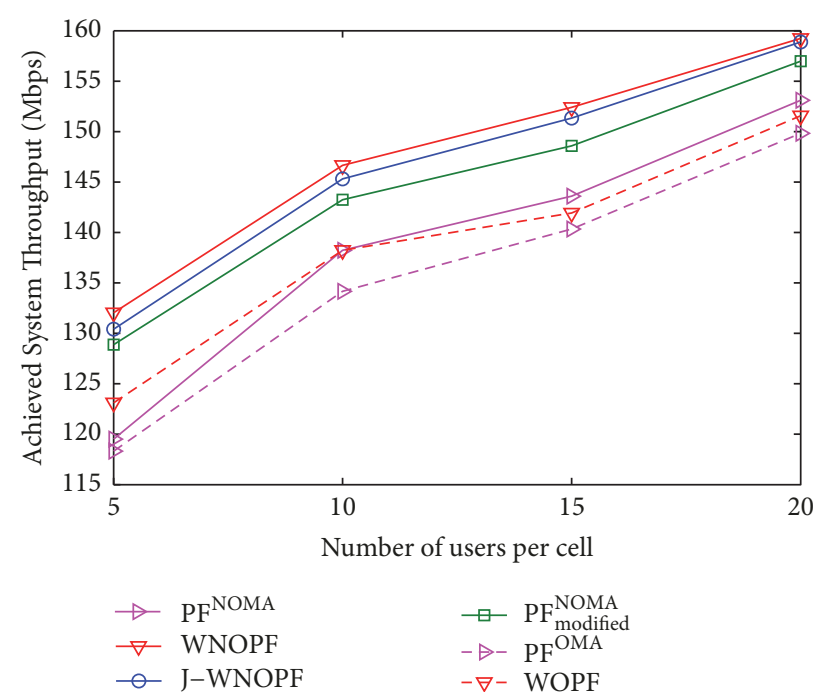

FIGURE 3: System throughput achieved with the proposed scheduling schemes versus number of users per cell.

Figure 3 also shows an improved performance of the proposed metrics when compared to the modified PF scheduling metric $\mathrm{PF}_{\text {modified }}^{\mathrm{NOMA}}$ described in [17]. Although they both consider the current assignment in their metric calculation, they still differ by the fact that the proposed weighted metrics target a higher rate compared to the rate previously achieved, therefore tending to increase the achieved user rate in every slot.

When the proposed scheduling metrics are applied in an OMA context, WOPF provides higher throughputs than $\mathrm{PF}^{\mathrm{OMA}}$, due to the same reason why WNOPF outperforms $\mathrm{PF}^{\mathrm{NOMA}}$. Figure 3 also shows a significant performance gain achieved by NOMA over OMA. All weighted scheduling metrics applying NOMA outperform the simulated metrics based on OMA, including WOPF. This gain is due to the efficient nonorthogonal multiplexing of users. It should also be noted that the gain achieved by WNOPF over $\mathrm{PF}^{\mathrm{NOMA}}$ is greater than the one achieved by WOPF over OPF: combining fair weights with NOMA definitely yields the best performance.

Long-term fairness is an important performance indicator for the allocation process. Figure 4 shows this metric as a function of the number of users per cell. Long-term fairness is improved when fair weights are introduced, independently of the access technique (OMA or NOMA). The reason is that, when aiming to enhance fairness in every scheduling slot, long-term fairness is enhanced accordingly. Again, in terms of fairness, the proposed weighted metrics outperform the modified PF metric [17], $\mathrm{PF}_{\text {modified. }}^{\mathrm{NOMA}}$. This is due to the fact that WNOPF and J-WNOPF not only consider the current rate assignment but also tend to minimize the rate gap among scheduled users in every channel realization, thus maximizing fairness among them.

Figure 5 shows the achieved system throughput as a function of the number of subbands $S$, for 15 users per cell. We can see that the proposed weighted metrics outperform

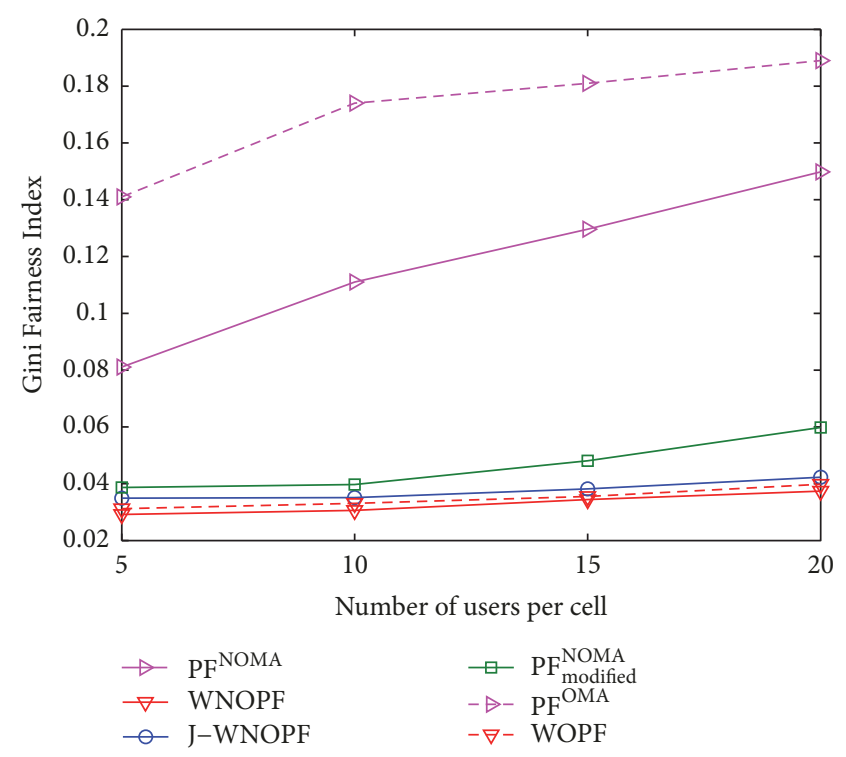

FIGURE 4: Gini fairness index of the proposed scheduling schemes versus number of users per cell.

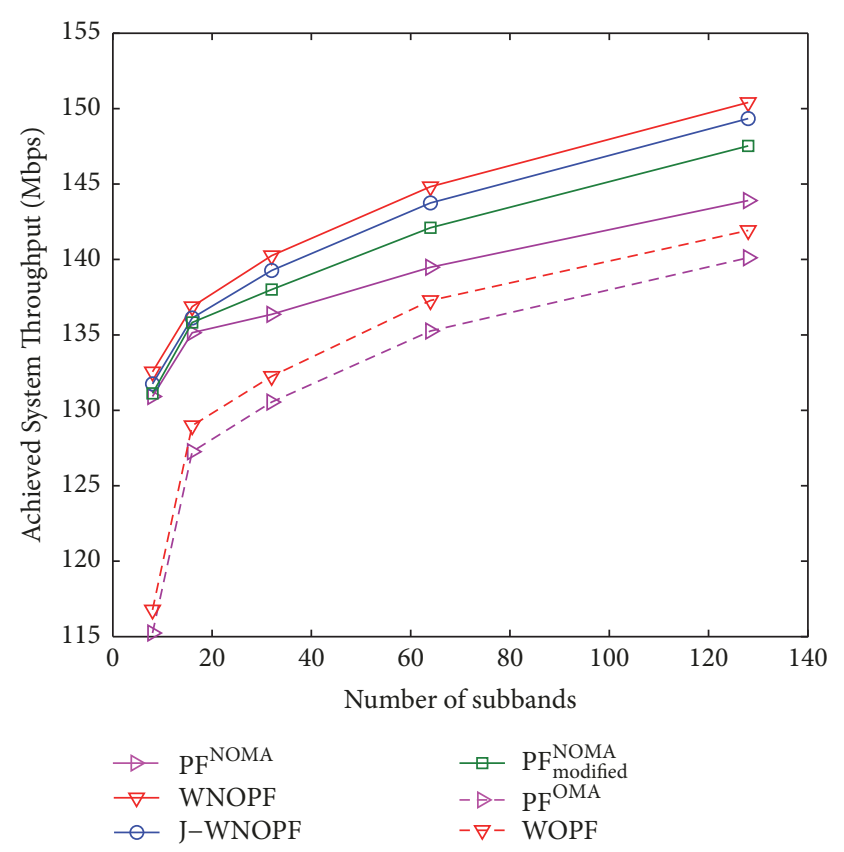

Figure 5: Achieved system throughput versus $S$, for $K=15$.

the conventional PF scheduling scheme, for both access techniques OMA and NOMA, even when the number of subbands is limited. It is also shown that the proposed weighted metrics outperform the modified PF metric [17], $\mathrm{PF}_{\text {modified }}^{\mathrm{NOMA}}$.

Since WNOPF proves to give better performance than JWNOPF, in terms of system capacity and fairness, J-WNOPF will not be considered in the subsequent results.

Since one of the main focuses of this study is to achieve short-term fairness, the proposed techniques should be compared based on the time required to achieve the final 


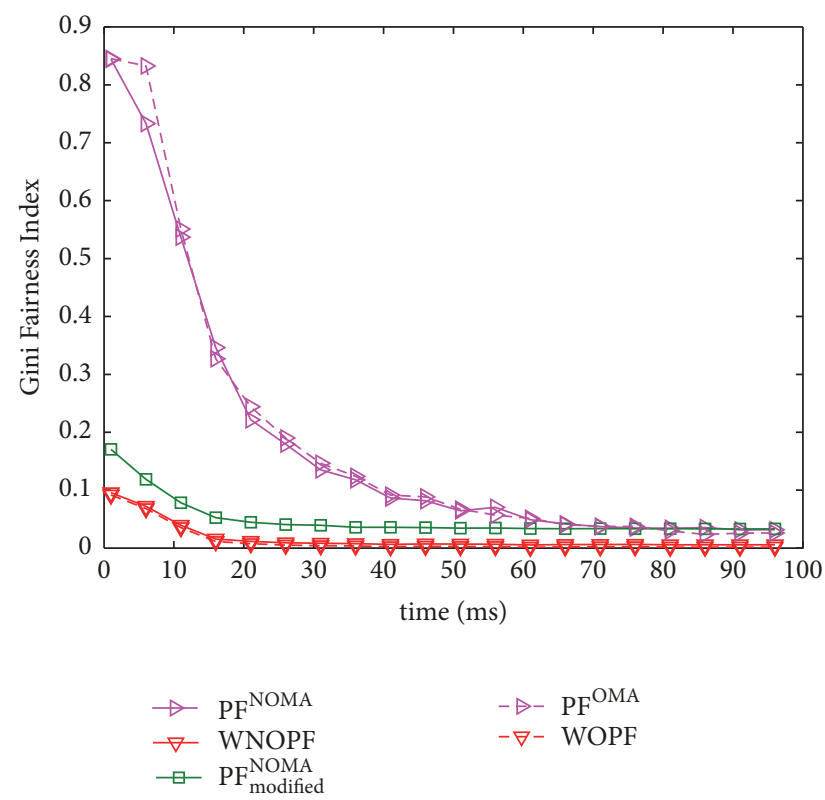

FIGURE 6: Gini fairness index versus scheduling time index $t$.

fairness level. Figure 6 shows the Gini fairness index versus the scheduling time index $t$. The proposed weighted metric WNOPF achieves high fairness from the beginning of the allocation process and converges to the highest level of fairness (lowest value of index $G=0.0013$ ) in a limited number of allocation steps or time slots. On the contrary, $\mathrm{PF}^{\mathrm{NOMA}}$ shows unfairness among users for a much longer time. Weighted metrics not only show faster convergence to a high fairness level but also give a lower Gini indicator at the end of the window length, when compared to conventional $\mathrm{PF}^{\mathrm{NOMA}}$. Figure 6 shows also that the proposed weighted metric WNOPF still outperforms the modified PF method proposed in [14].

In order to assess the QoE achieved by the proposed scheduling schemes, we evaluate the time required for each user to be served for the first time, referred to as the rate latency, as well as the variations of its achieved rate over time. For this purpose, Figure 7 shows the achieved rate versus time for the user experiencing the largest rate latency, for the different scheduling schemes.

When the conventional $\mathrm{PF}^{\mathrm{NOMA}}$ is used, no rate is provided for this user, for the first five scheduling slots. In addition, large rate fluctuations are observed through time. In contrast, when weighted metrics and a special treatment of the first time slot are considered, a nonzero rate is assigned for the least privileged users from the first scheduling slot and remains stable for all the following slots. This behavior results from the fact that, at the beginning of the scheduling process (first scheduling slot), historical rates are set to zero, and $\mathrm{PF}^{\mathrm{NOMA}}$ uses only instantaneous achievable throughputs to choose the best candidate user set. Therefore, users experiencing bad channel conditions have a low chance to be chosen. The corresponding achieved data rates are then equal to zero. On the other side, using the

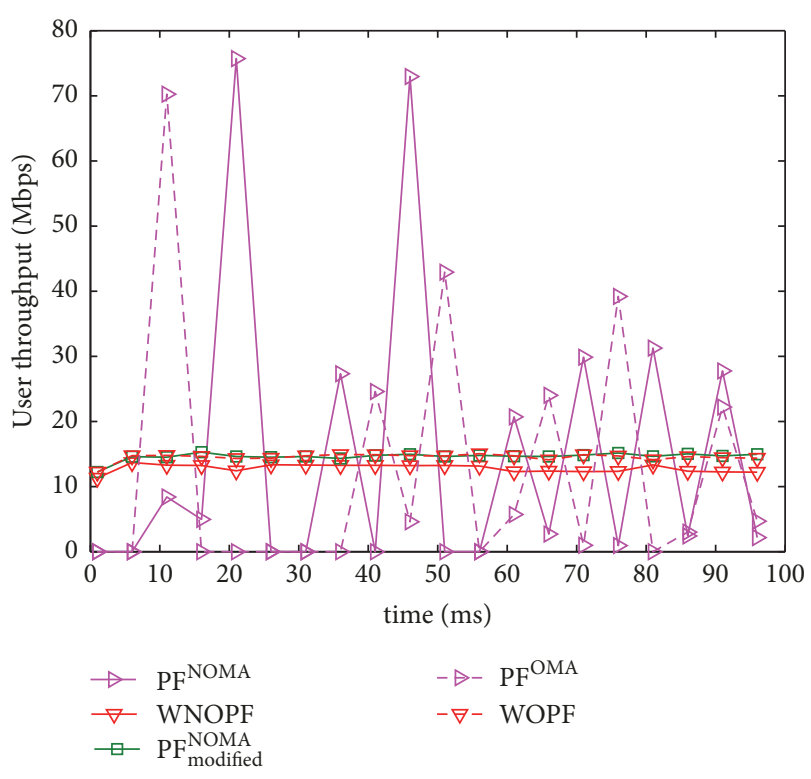

FIGURE 7: User throughput versus time for NOMA-based scheduling schemes.

proposed scheduling, the treatment of the first scheduling slot is conducted differently and users are chosen depending on their actual rates (measured during the actual scheduling period). In this case, zero rates are eliminated. Hence, latency is greatly reduced.

For the next scheduling slots, historical rates are taken into account. For $\mathrm{PF}^{\mathrm{NOMA}}$, users experiencing a large $T_{k}(t)$ have less chance to be chosen and may not be chosen at all. In this case, the use of buffering becomes mandatory and the size of the buffer should be chosen adequately to prevent overflow when peak rates occur, as a result of a high achieved throughput (high $R_{s, k}(t)$ ). Based on calculation, the average size of the buffer should be around $110 \mathrm{Mbit}$, for the simulation case at hand. However, in the case of the weighted proposed metrics, buffering is not needed, since only small variations between user data rates are observed, and a better QoE is achieved. Similar performance improvement is obtained for the orthogonal case in the same aforementioned conditions.

Finally, we have analyzed the effect of the proposed scheduling scheme on the cell-edge user throughput in Figure 8. Again, the proposed weighted metrics outperform the conventional PF scheduling scheme for both access techniques, OMA and NOMA. In addition, WNOPF shows the best performance. Therefore, we can state that the incorporation of fair weights with a NOMA-based system proves to be the best combination.

In order to evaluate the performance of the proposed weighted metrics when premium services are considered, Tables 1 and 2 show the Gini fairness index values for two different scenarios, where three levels of services are requested: basic, silver, and gold. The number of users per group is set to 5 . 


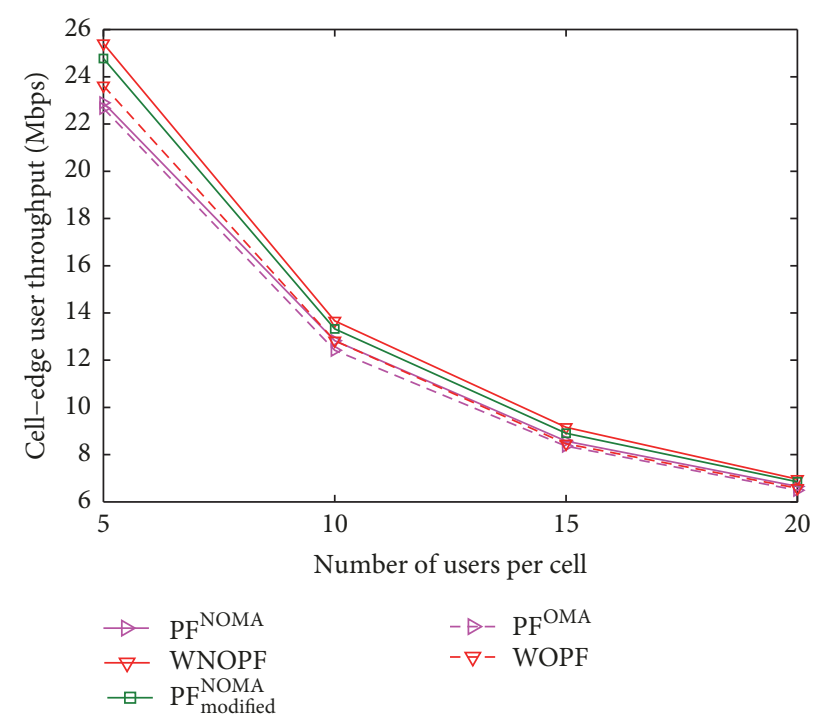

FIGURE 8: Cell-edge user throughput versus number of users per cell.

TABLE 1: Gini fairness index and data rate achieved per group for Scenario 1 (100\% success).

\begin{tabular}{lcc}
\hline Service & Gini fairness index & $\begin{array}{c}\text { Achieved data rate per } \\
\text { group (Mbps) }\end{array}$ \\
\hline Basic & 0.0491 & 25.7 \\
Silver & 0.0724 & 51 \\
Gold & 0.0042 & 76.3 \\
\hline
\end{tabular}

TABLE 2: Gini fairness index and data rate achieved per group for Scenario 2 (no success).

\begin{tabular}{lcc}
\hline Service & Gini fairness index & $\begin{array}{c}\text { Achieved data rate per } \\
\text { group (Mbps) }\end{array}$ \\
\hline Basic & 0.0522 & 30.2 \\
Silver & 0.0613 & 49.6 \\
Gold & 0.0049 & 75.2 \\
\hline
\end{tabular}

Scenario 1. The corresponding data rates of the three levels are set to $5 \mathrm{Mbps}, 10 \mathrm{Mbps}$, and $15 \mathrm{Mbps}$, respectively.

Scenario 2. The corresponding data rates of the three levels are set to $10 \mathrm{Mbps}$, $20 \mathrm{Mbps}$, and $30 \mathrm{Mbps}$, respectively.

The objective behind adding such scenario is to show the behavior of the system regarding user fairness when the target data rate is set to be relatively very high, thus when target data rate is not met.

In Scenario 1, all users succeed in reaching their requested service data rates, and results of Table 1 show a high level of fairness achieved among users requesting the same service. However, when Scenario 2 is applied, no success could be obtained but fairness is still maintained among users. This analysis can show that the proposed algorithm tries to boost and improve the data rates of all users in such a way to still maintain an improved performance at the level of user fairness even when the target data rate is not met.
7.3. Computational Complexity. With the aim of assessing the implementation feasibility of the different proposed schedulers, we measured the computational load of the main allocation techniques to be integrated at the BS.

From a complexity point of view, the proposed scheduling metric WNOPF differs from the conventional PF metric in the weight calculation. For a number of users per subband limited to 2 in NOMA, the number of candidates per subband is $\left(\begin{array}{l}1 \\ K\end{array}\right)+\left(\begin{array}{l}2 \\ K\end{array}\right)$. When listing the operations of the proposed allocation technique, we obtain that the proposed metric WNOPF increases the PF computational load by $(26 / 3) K S+$ $S(\simeq O(K S))$ multiplications and $-K^{3} S+(3 / 2) K^{2} S^{2}-$ $(4 / 6) K^{2} S-(3 / 6) K S\left(\simeq O\left((3 / 2) K^{2} S^{2}-K^{3} S\right)\right)$ additions.

In order to compute the PF metric for a candidate user set containing only 1 user, $4+S$ multiplications and $1+(3 / 2) S$ additions are needed. For each candidate user set containing 2 multiplexed users, $13+2 S$ multiplications and $6+3 S$ additions are required.

By taking account of the calculations of the terms $h^{-2 \alpha}, h^{2}$, and $h^{2} /\left(N_{0} B / S\right)$ performed at the beginning of the allocation process, the classical NOMA PF requires a total of $3 K S+$ $C_{K}^{1} S(4+S)+C_{K}^{2} S(13+2 S)$ multiplications, which are equal to $K^{2} S^{2}+(1 / 2) K S+(13 / 2) K^{2} S\left(\simeq O\left(K^{2} S^{2}\right)\right)$ and $C_{K}^{1} S(1+$ $3 S / 2)+C_{K}^{2} S(6+3 S)$ additions, which are equal to $(3 / 2) K^{2} S^{2}+$ $(1 / 2) K S+(13 / 2) K^{2} S\left(\simeq O\left(K^{2} S^{2}\right)\right)$. Therefore, we can see that the increase in the number of multiplications is minor in comparison with that of the conventional PF, while the number of additions is almost doubled.

\section{Conclusion}

In this paper, we have proposed new weighted scheduling schemes for both NOMA and OMA multiplexing techniques. They target maximizing fairness among users, while improving the achieved capacity. Several fair weights designs have been investigated. Simulation results show that the proposed schemes allow a significant increase in the total user throughput and the long-term fairness, when compared to OMA and classic NOMA-based PF scheduler. Combining NOMA with fair weights shows the best performance. Furthermore, the proposed weighted techniques achieve a high level of fairness within each scheduling slot, which improves the QoE of each user. In addition, the proposed weighted metrics give the possibility of delivering different levels of QoS, which can be very useful for certain applications. The study conducted here with two scheduled users per subband can be easily adapted to a larger number of users. Initially developed for single-antenna systems, the proposed scheduling technique can be extended to support multiantenna systems. Such an extension could be performed using our previous work in [32]. We are currently undergoing further research to reduce the complexity of the PF scheduler by introducing an iterative allocation scheme.

\section{Data Availability}

The data used to support the findings of this study are available from the corresponding author upon request. 


\section{Conflicts of Interest}

The authors declare that they have no conflicts of interest.

\section{Acknowledgments}

Part of this work has been performed in the framework of the Horizon 2020 project FANTASTIC-5G (ICT-671660), which is partly funded by the European Union. The authors would like to acknowledge the contributions of their colleagues in FANTASTIC-5G. This work has also been funded with support from the Lebanese University and the FrenchLebanese CEDRE program.

\section{References}

[1] 3GPP TS36.300, Evolved Universal Terrestrial Radio Access (E-UTRA) and Evolved Universal Terrestrial Radio Access Network (E-UTRAN); Overall description.

[2] 3GPP TR36.913 (V8.0.0), 3GPP, and TSG RAN, Requirements for further advancements for E-UTRA (LTE-Advanced), 2008.

[3] 3GPP TR36.814 (V9.0.0), Further Advancements for E-UTRA Physical Layer Aspects, 2010.

[4] "Ericsson Mobility Report, on the pulse of the networked society," https://www.ericsson.com/assets/local/mobility-report/ documents/2017/ericsson-mobility-report-june-2017.pdf, June 2017.

[5] Y. Chen, A. Bayesteh, Y. Wu et al., "Toward the standardization of non-orthogonal multiple access for next generation wireless networks," IEEE Communications Magazine, vol. 56, no. 3, pp. 19-27, 2018.

[6] G. Caire and S. Shamai, "On the achievable throughput of a multiantenna Gaussian broadcast channel," IEEE Transactions on Information Theory, vol. 49, no. 7, pp. 1691-1706, 2003.

[7] T. Takeda and K. Higuchi, "Enhanced user fairness using nonorthogonal access with SIC in cellular uplink," in Proceedings of the IEEE 74th Vehicular Technology Conference, VTC Fall 2011, September 2011.

[8] K. Higuchi and Y. Kishiyama, "Non-orthogonal access with random beamforming and intra-beam SIC for cellular mimo downlink," in Proceedings of the IEEE 78th Vehicular Technology Conference (VTC '13), pp. 1-5, Las Vegas, Nev, USA, September 2013.

[9] N. Otao, Y. Kishiyama, and K. Higuchi, "Performance of nonorthogonal access with SIC in cellular downlink using proportional fair-based resource allocation," in Proceedings of the 2012 9th International Symposium on Wireless Communication Systems, ISWCS 2012, pp. 476-480, August 2012.

[10] Y. Saito, Y. Kishiyama, A. Benjebbour, T. Nakamura, A. Li, and K. Higuchi, "Non-orthogonal multiple access (NOMA) for cellular future radio access," in Proceedings of the IEEE 77th Vehicular Technology Conference (VTC '13), pp. 1-5, Dresden, Germany, June 2013.

[11] J. Umehara, Y. Kishiyama, and K. Higuchi, "Enhancing user fairness in non-orthogonal access with successive interference cancellation for cellular downlink," in Proceedings of the IEEE 14th International Conference on Communication Systems (ICCS '12), pp. 324-328, Singapore, November 2012.

[12] Sharp corporation, "Evolving RAN towards Rel-12 and beyond, RWS-120039," in Proceedings of the 3GPP Workshop on Release 12 Onward, Ljubljana, Slovenia, 2012.
[13] H. Xing, Y. Liu, A. Nallanathan, Z. Ding, and H. V. Poor, "Optimal throughput fairness trade-offs for downlink nonorthogonal multiple access over fading channels," IEEE Transactions on Wireless Communications, pp. 1-1, 2017.

[14] Q. Pham and W. Hwang, " $\alpha$-Fair resource allocation in nonorthogonal multiple access systems," IET Communications, vol. 12, no. 2, pp. 179-183, 2018.

[15] J. Schaepperle, "Throughput of a wireless cell using superposition based multiple-access with optimized scheduling," in Proceedings of the 2010 IEEE 21st International Symposium on Personal Indoor and Mobile Radio Communications, PIMRC 2010, pp. 212-217, September 2010.

[16] M.-R. Hojeij, J. Farah, C. A. Nour, and C. Douillard, "New optimal and suboptimal resource allocation techniques for downlink non-orthogonal multiple access," Wireless Personal Communications, vol. 87, no. 3, pp. 837-867, 2016.

[17] E. Okamoto, "An improved proportional fair scheduling in downlink non-orthogonal multiple access system," in Proceedings of the 82nd IEEE Vehicular Technology Conference, VTC Fall 2015, September 2015.

[18] M. Mehrjoo, M. K. Awad, M. Dianati, and X. S. Shen, "Design of fair weights for heterogeneous traffic scheduling in multichannel wireless networks," IEEE Transactions on Communications, vol. 58, no. 10, pp. 2892-2902, 2010.

[19] C. Gueguen and S. Baey, "Compensated proportional fair scheduling in multiuser OFDM wireless networks," in Proceedings of the IEEE International Conference on Wireless \& Mobile Computing, Networking \& Communications, 2008.

[20] C. Yang, W. Wang, Y. Qian, and X. Zhang, "A weighted proportional fair scheduling to maximize best-effort service utility in multicell network," in Proceedings of the 2008 IEEE 19th International Symposium on Personal, Indoor and Mobile Radio Communications, PIMRC, September 2008.

[21] D. Tse and P. Viswanath, Fundamentals of Wireless Communication, Cambridge University Press, Cambridge, UK, 2005.

[22] S. Tomida and K. Higuchi, "Non-orthogonal access with SIC in cellular downlink for user fairness enhancement," in Proceedings of the 2011 International Symposium on Intelligent Signal Processing and Communications Systems (ISPACS 2011), pp. 1-6, Chiang Mai, Thailand, December 2011.

[23] A. Benjebbour, Y. Saito, Y. Kishiyama, A. Li, A. Harada, and T. Nakamura, "Concept and practical considerations of nonorthogonal multiple access (NOMA) for future radio access," in Proceedings of the 2013 21st International Symposium on Intelligent Signal Processing and Communication Systems, ISPACS 2013, pp. 770-774, November 2013.

[24] B. Kimy, S. Lim, H. Kim et al., "Non-orthogonal multiple access in a downlink multiuser beamforming system," in Proceedings of the 2013 IEEE Military Communications Conference, MILCOM 2013, pp. 1278-1283, November 2013.

[25] Y. Saito, A. Benjebbour, Y. Kishiyama, and T. Nakamura, "System-level performance evaluation of downlink nonorthogonal multiple access (NOMA)," in Proceedings of the 2013 IEEE 24th Annual International Symposium on Personal, Indoor, and Mobile Radio Communications, PIMRC, pp. 611-615, September 2013.

[26] F. P. Kelly, A. K. Maulloo, and D. Tan, "Rate control for communication networks: shadow prices, proportional fairness and stability," Journal of the Operational Research Society, vol. 49, no. 3, pp. 206-217, 1997.

[27] P. Viswanath, D. N. Tse, and R. Laroia, "Opportunistic beamforming using dumb antennas," Institute of Electrical and 
Electronics Engineers Transactions on Information Theory, vol. 48, no. 6, pp. 1277-1294, 2002.

[28] M. Kountouris and D. Gesbert, "Memory-based opportunistic multi-user beamforming," in Proceedings of the International Symposium on Information Theory, ISIT 2005, pp. 1426-1430, Adelaide, Australia, September 2005.

[29] 3GPP and TR25-814 (V7.1.0), Physical Layer Aspects for Evolved UTRA, 2006.

[30] A. Benjebbovu, A. Li, Y. Saito, Y. Kishiyama, A. Harada, and T. Nakamura, "System-level performance of downlink NOMA for future LTE enhancements," in Proceedings of the IEEE Globecom Workshops, pp. 66-70, Atlanta, Ga, USA, December 2013.

[31] M. Dianati, X. Shen, and S. Naik, "A new fairness index for radio resource allocation in wireless networks," in Proceedings of the 2005 IEEE Wireless Communications and Networking Conference, WCNC 2005: Broadband Wirelss for the Masses-Ready for Take-off, pp. 712-717, March 2005.

[32] M.-J. Youssef, J. Farah, C. A. Nour, and C. Douillard, "Waterfilling-based resource allocation techniques in downlink Non-Orthogonal Multiple Access (NOMA) with single-user MIMO," in Proceedings of the 2017 IEEE Symposium on Computers and Communications, ISCC 2017, pp. 499-506, July 2017. 


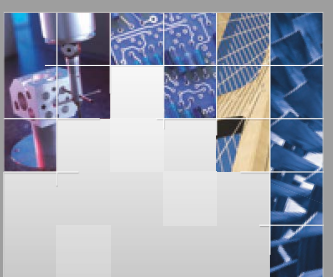

\section{Enfincering}
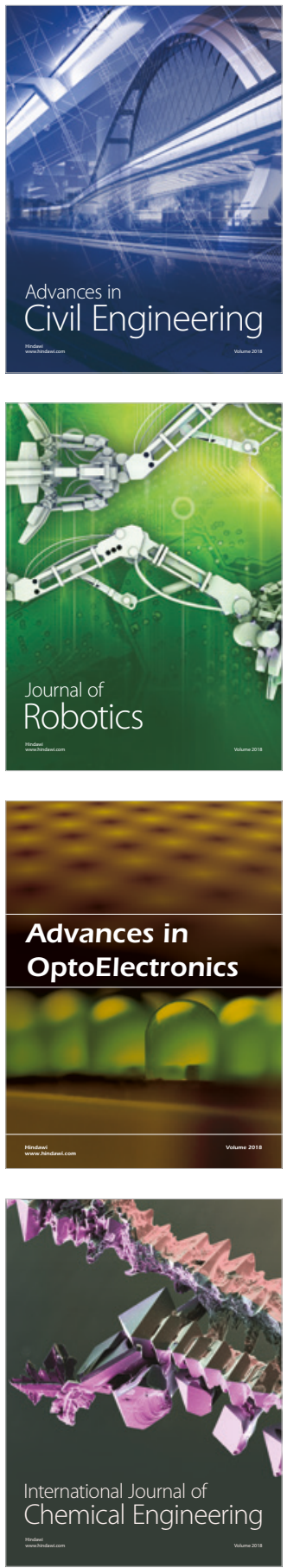

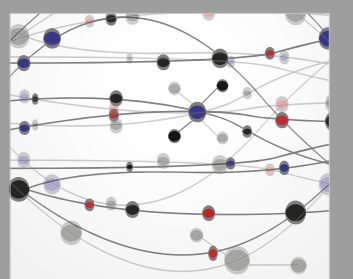

\section{Rotating \\ Machinery}

The Scientific World Journal

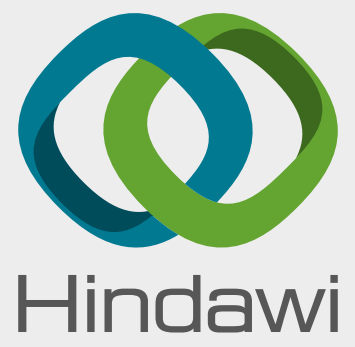

Submit your manuscripts at

www.hindawi.com
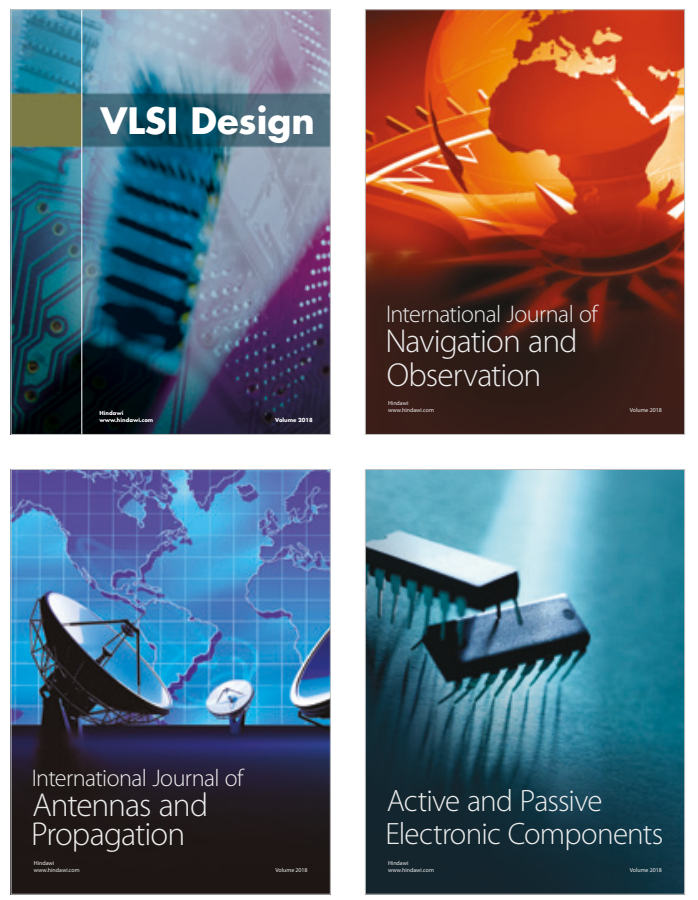
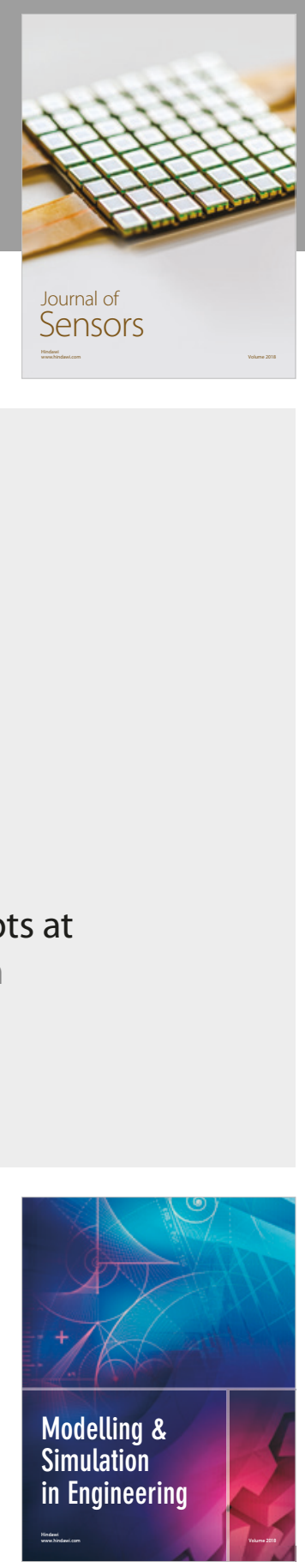

\section{Advances \\ Multimedia}
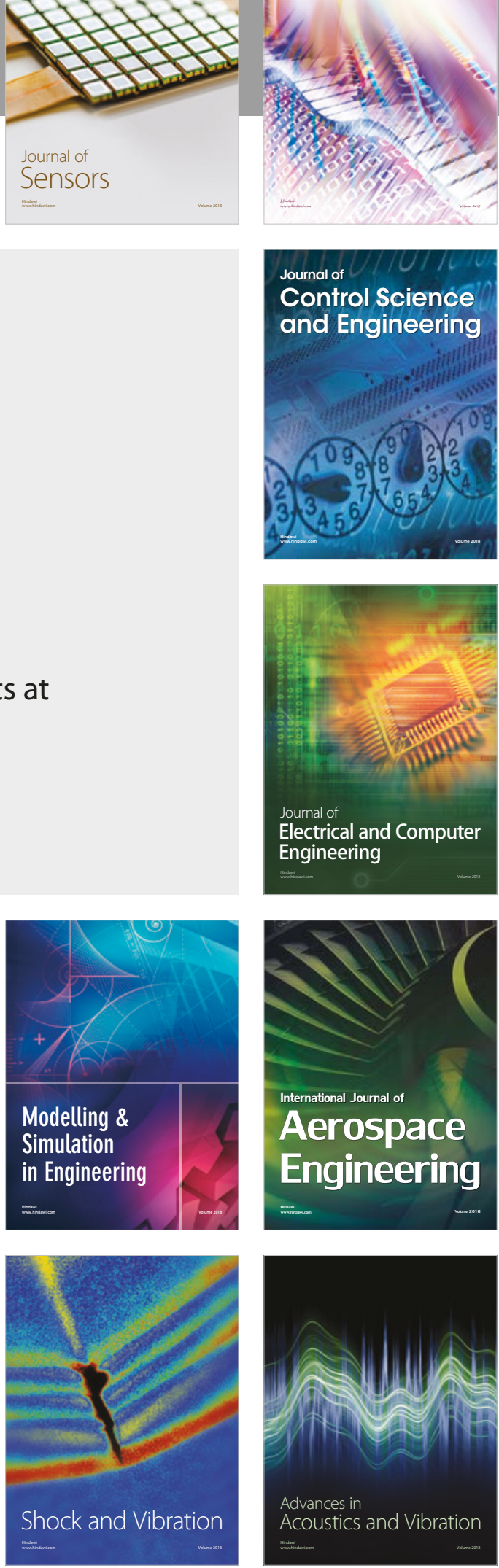